\title{
Trauma and orthopaedic surgeons
}

The relationship between elective orthopaedic surgery and the acute management of musculoskeletal trauma has never been comfortable: for many reasons, these two aspects of care of the same system are not easy to combine. In some countries these are distinct specialties, which creates other problems. In the United Kingdom an experiment in which the Birmingham Accident Hospital provided all-system care for trauma did not become generally accepted.

It can be argued that injury to one region or organ should be managed by experts in that field, despite the problems created by conflicting priorities and loss of overall control. In most specialties, elective and acute care can be relatively easily combined, but in orthopaedics the sheer number of trauma patients and the recent rapid division into subspecialties has created new difficulties.

The two main concerns are the care of critically ill patients with multiple injuries, and the treatment of increasing numbers of cases of isolated trauma to bones, joints and soft tissues. These are closely related problems, but are rarely considered together. There can be no clear general answer which will be applicable throughout the world: local conditions vary too much in terms of the pattern of trauma, population density, transport facilities and the level of available expertise.

In developed countries, the patient-related factors now include an ageing population, a boom in athletic leisure pursuits, and high expectations of complete recovery, while financial and political factors also have an influence. Countries with comprehensive health services are finding it increasingly difficult and expensive to provide the treat- ments made possible by technical advances in surgery. Patients (and some surgeons) will often opt for the 'latest' method, even if it has been devised for expert use only, and for a particular type of problem. Fashions come and go; methods which have been devised for limited indications become widely adopted.

An isolated displaced tibial fracture provides one example: there have been successive waves of popularity for plate and screw fixation, for external fixation, and for intramedullary nails, first reamed and now unreamed. These methods all need special skills; but all produce their own set of complications, especially during successive learning curves. In the past, manipulation and plaster provided many satisfactory results and this may still be a viable option. It is unfortunate that the experience needed to select this simple treatment and the skill to perform it well are being lost in favour of high technology.

Because of the wide variation in practice in countries with differing traditions, population density and patient expectation it is impossible to provide an overall view for our world-wide readership. To allow a constructive debate, it seemed best to have contributions from a number of surgeons with experience in trauma management. In this issue, Charles Court-Brown and Margaret McQueen give their forthright views on the state of the primary care of musculoskeletal injuries in the United Kingdom. Future editorials are being invited from a number of other countries.

PHILIP FULFORD EDITOR

\section{Trauma management in the United Kingdom}

The organisation and management of musculoskeletal injuries in the United Kingdom require fundamental change. In recent years, orthopaedic surgery has become increasingly sub-specialised: more surgeons are choosing to concentrate

C. Court-Brown, FRCS Ed Orth, Consultant Orthopaedic Surgeon M. M. McQueen, FRCS Ed Orth, Consultant Orthopaedic Surgeon The Royal Infirmary of Edinburgh, Lauriston Place, Edinburgh EH3 9YW, UK.

(C)1997 British Editorial Society of Bone and Joint Surgery 0301-620X/97/17020\$2.00

J Bone Joint Surg [Br] 1997;79-B:1-3. on elective disciplines or single regions of the body. Many remain administratively responsible and on call for acute trauma, but lack time and interest to keep up with modern advances in its management. This applies both to major life or limb threatening injuries and to common isolated injuries outside their chosen elective field.

We have recently tried to assess the specialty interests of UK orthopaedic surgeons by telephoning representatives from many areas. We asked each of them for a maximum of two specialty interests, not to provide a complete analysis, but to record special interest in musculoskeletal trauma. Table I shows that only $8.3 \%$ of surgeons reported a major 
interest in trauma and most of these also had another elective discipline. Only about a dozen surgeons in the United Kingdom practice primary and secondary trauma surgery alone, but about $80 \%$ of those we surveyed were on call for trauma. This is a remarkable imbalance, indicating a strong bias towards elective and late post-traumatic reconstructive surgery. Patients with musculoskeletal injuries often receive inadequate primary care and therefore frequently require secondary reconstruction. This is expensive, time consuming and difficult; it also leads to litigation.

Table I. The frequencies of the declared orthopaedic specialties, including those surgeons who consider themselves to be general orthopaedic surgeons

\begin{tabular}{ll}
\hline & Per cent \\
\hline General orthopaedic & 20.7 \\
Arthroplasty including rheumatoid & 15.7 \\
Spinal & 11.5 \\
Paediatric & 11.3 \\
Knee & 9.7 \\
Upper limb & 9.1 \\
Trauma & 8.3 \\
Hand & 6.7 \\
Foot & 3.0 \\
Oncology & 2.5 \\
Amputation and prosthetics & 0.6 \\
Others & 0.8
\end{tabular}

A recent census by the British Orthopaedic Association ${ }^{1}$ confirmed our findings: only $6.7 \%$ of orthopaedic surgeons had a specialist interest in trauma and this proportion decreased with age. Only $2.7 \%$ of surgeons over 55 years of age expressed such an interest. Many professors of orthopaedic surgery are not on call for trauma, further indicating that political weight and the emphasis of training tend to be heavily skewed towards elective work. A change in this balance is unlikely when very few senior and influential clinicians have the necessary specialist knowledge and experience of trauma management.

Another review by the British Orthopaedic Association found that in the UK, with a population of approximately 55 million, there are about 900000 musculoskeletal injuries each year. Over 100000 of these patients are left with a significant disability. ${ }^{2}$ Capewell, ${ }^{3}$ from Scotland, supported by figures for the whole of the UK, has shown that emergency admissions in all specialties are increasing by $5 \%$ per year compared with an average increase of $1 \%$ per year for elective cases. It is evident that the trauma "problem' is increasing at a rate which will eventually jeopardise the proper provision of elective surgery.

As far as we are aware only two centres in the UK have completely separated the management of acute injuries from elective surgery: these are Edinburgh and Oxford, where trauma units are staffed full-time by orthopaedic traumatologists. In Edinburgh, this recent subdivision has enabled us to show that $60 \%$ of all adult orthopaedic management is performed for trauma. Even then, a proportion of the work classed as elective consists of posttraumatic reconstruction. The size of the whole problem of trauma care is therefore even greater than has been previously recognised.

Two areas of the primary treatment of trauma need consideration. These are the management of severely and multiply-injured patients and the much larger problem of isolated musculoskeletal injury with no other system involved. Recent interest in the UK has centred on the care of multiply-injured patients. The Irving report of the Royal College of Surgeons ${ }^{4}$ showed major deficiencies: seriously injured patients were being treated by inexperienced junior staff. In response to this report a specially financed trauma centre was established in the North Staffordshire Royal Infirmary at Stoke-on-Trent in an attempt to investigate the relevance of such 'American style' Level 1 Trauma centres. The results were compared with those of two other regions which provided the standard facilities of District General Hospitals but included on-site neurosurgery. It was concluded that patients gained no significant benefit from being treated in the trauma centre, but the methodology of the trial had some serious flaws and it seems doubtful whether the results are meaningful. Despite this, the failure to prove that any significant advantage was derived from a relatively expensive trauma centre will undoubtedly be used as evidence that such centres are inappropriate and unnecessary in the United Kingdom.

This seems somewhat depressing. The management of very severely injured patients should be important to any civilised society, and high standards have been set at a number of centres around the world. ${ }^{5}$ However, such multiply-injured patients form only about $1 \%$ of the musculoskeletal trauma admitted to UK hospitals. There is a danger that both surgeons and managers will be tempted to assume that the problem of trauma is solved when adequate protocols and treatments have been established for seriously injured patients. Deaths due to road traffic accident have decreased in the last 10 to 15 years and with improved road and vehicle engineering, advertising campaigns and safety laws this trend will probably continue.

The numerically much greater, but less emotive problem, of isolated musculoskeletal injuries is increasing with the greater longevity of the population, and the boom in leisure time athletic activity. Not unreasonably, these patients now expect good treatment and full restoration to normality, but their initial management is often undertaken by unsupervised junior doctors. $^{6}$ All too often these junior staff have not had appropriate training and experience; they tend to create future problems by practising inadequate primary care.

In our opinion, the best way to provide a system which can cope with the management of all trauma problems, 
including those of the severely injured, is better organisation of the management of isolated musculoskeletal injuries. At present, specialist surgeons in this field spend much time and effort in treating the complications of inadequate primary management. This is expensive, wasteful of resources and often compromises the eventual outcome. It would not be too difficult to improve trauma management in the United Kingdom, but the interest and will of the profession are essential. The larger central hospitals should each have surgeons employed as full-time traumatologists to perform both primary and reconstructive trauma surgery. In smaller hospitals this is not possible, but more surgeons should be encouraged to make trauma a special interest and accept referral of difficult cases from consultants with other elective interests.

Financial factors, public and private, create more difficulty. Trauma surgeons require time and better resources. They are often expected to undertake the management of both multiple and isolated injuries as well as maintaining an elective practice. This is barely possible and it is hardly surprising that many surgeons choose the option of delegating the management of trauma to less experienced surgeons. Improved trauma resources will also benefit elective orthopaedic surgery, since these surgeons will be able to concentrate on their chosen specialties. Unfortunately these ideas have little attraction to hospital managers driven by political expediency and often pre-occupied by waiting times for elective operations.

There is little understanding of the social and financial implications of a poor quality of trauma care, and it is important that orthopaedic surgeons should change their approach to this, the largest of their sub-specialties. If this is not done, the public, the politicians and the legal profession will begin to ask even more searching questions about the quality of some of our current management.

C. COURT-BROWN M. M. MCQUEEN

\section{REFERENCES}

1. Philips H. The SAC and training in trauma. Brit Orthop News Summer 1996.

2. British Orthopaedic Association. The management of skeletal trauma in the United Kingdom. London: BOA, 1992.

3. Capewell $\mathbf{S}$. The continuing rise in emergency admissions. BMJ 1996;312:991-2.

4. Royal College of Surgeons of England. The management of patients with major injuries. London: RCS, 1988.

5. Tscherne H, Regel G. Care of the polytraumatised patient. $J$ Bone Joint Surg $[\mathrm{Br}]$ 1996;78-B:840-52.

6. Court-Brown CM, McQueen MM, Christie J. OxDONS syndrome. BMJ 1996;312:1927. 